\title{
Correlation of EGFR gene amplification with invasion and metastasis of non-small cell lung
} cancer

\author{
X.F. Jia ${ }^{1}$, J. Li ${ }^{1}$, H.B. Zhao ${ }^{1}$, J. Liu ${ }^{2}$ and J.J. Liu ${ }^{3}$ \\ ${ }^{1}$ Department of Oncology, The First People's Hospital of Jining City, \\ Jining, China \\ ${ }^{2}$ Department of Oncology, Shandong Cancer Hospital, Jinan, China \\ ${ }^{3}$ Department of Respiration, The First People's Hospital of Jining City, \\ Jining, China \\ Corresponding author: H.B. Zhao \\ E-mail: HaiboZhao0537@163.com
}

Genet. Mol. Res. 14 (3): 11006-11012 (2015)

Received January 14, 2015

Accepted May 25, 2015

Published September 21, 2015

DOI http://dx.doi.org/10.4238/2015.September.21.13

ABSTRACT. The aim of this study was to explore epidermal growth
factor receptor (EGFR) gene amplification and its relationship
with cancer invasion and metastasis in non-small cell lung cancer
(NSCLC). EGFR amplification in 45 patients with NSCLC and 15
subjects with normal lung tissues was detected by fluorescence in
situ hybridization. The relationship between $E G F R$ amplification and
the clinicopathologic features of NSCLC was analyzed. EGFR gene
amplifications were identified in 2 of 15 normal lung tissues $(13.33 \%)$
and in 29 of 45 NSCLCs $(64.44 \%)$. Patients $<60$ years had a $66.67 \%$
$E G F R$ amplification rate, while patients $\geq 60$ years had a rate of $62.50 \%$.
The $E G F R$ amplification rates in male and female patients were $64.0 \%$
$(16 / 25)$ and $65.0 \%(13 / 20)$, respectively. Pathologically, the $E G F R$
amplification rate of patients with squamous cell carcinoma was $56.52 \%$
$(13 / 23)$, and with adenocarcinoma was $72.72 \%(16 / 22)$. The $E G F R$
amplification rate in NSCLCs with well-moderate differentiation was 
lower than in those with poor differentiation; $48.0 \%$ (12/25) vs $85.0 \%$ (17/20), respectively. Patients with lymph node metastasis had nearly double the amplification rate than those without metastasis; $90.0 \%$ (18/20) vs 44.0\% (11/25), respectively. The rate of EGFR amplification was significantly higher in NSCLC than in normal lung tissue (64.44 vs $13.33 \%, \mathrm{P}<0.05)$, and was not correlated with age or gender $(\mathrm{P}>0.05)$, but increased with clinical stage in NSCLCs $(\mathrm{P}<0.05)$. Overall, these studies found that the rate of $E G F R$ gene amplification was increased significantly in NSCLC and was closely related to lymphatic metastasis and TNM stage.

Key words: NSCLC; Metastasis; FISH; EGFR

\section{INTRODUCTION}

Lung cancer is one of the malignant tumors that poses the largest threat to human health and life, and is demonstrating rapidly increasing incidence and mortality. Non-small cell lung cancer (NSCLC) accounts for approximately $80-85 \%$ of the total lung cancer diagnosed. One-third of NSCLC cases appear locally advanced in the initial diagnosis, and the 5-year survival rate was found to be only about 15\% (Rivera, 2004; Parkin et al., 2005). Patients with advanced lung cancer that have lost the opportunity for surgical treatments are only able to rely on chemotherapy and radiotherapy for treatment options. Chemotherapy, however, is often accompanied by considerable side-effects because of its non-specificity. Currently, targeted biological therapies can kill tumor cells without damaging normal cells, and such treatment modes are becoming increasingly accepted by doctors and patients. Gefitinib is the most representative target drug, which acts on the epidermal growth factor receptor (EGFR) and has been recommended as the first-line treatment for NSCLC by the National Comprehensive Cancer Network (NCCN).

EGFR is the receptor for epithelial growth factor (EGF), and belongs to the ErbB receptor family, which mediates cell proliferation and signal transduction. EGFR is located on the surface of the cell membrane and can be activated after being combined with its ligand EGF and transforming growth factor $\alpha$ (TGF $\alpha$ ) (Sibilia et al., 2007). EGFR changes from a monomer to a dimer after activation. The EGFR dimer can activate an intracellular kinase pathway to regulate cell growth (Stinchcombe and Socinski, 2010). Previous studies have shown that abnormal expression of EGFR in many solid tumors as well as elevated EGFR expression were related to tumor cell proliferation, angiogenesis, invasion, metastasis, and the inhibition of apoptosis (Jorissen et al., 2003). Due to the important function of EGFR in tumor formation and development, targeted EGFR treatments have caught the attention of researchers worldwide. Considerable progress on lung cancer target drugs, focused on molecular markers and signaling pathways, has been attained in the past 10 years. Gefitinib and Erlotinib, as inhibitors of EGFR tyrosine kinase (EGFR-TKIs), have shown marked clinical efficacy on patients with NSCLC carrying EGFR mutations. Previous research has demonstrated that the sensitivity of patients with NSCLC to EGFR-TKIs was closely related to the state of the EGFR gene (Jeon et al., 2006; Tamura et al., 2008). In 2004, it was found that EGFR-TKIs presented different sensitivities to patients with different EGFR exon mutation status (Lynch et al., 2004; Paez et al., 2004). In addition, Cappuzzo et al. (2005) 
also found that EGFR-TKIs had a better curative effect in patients with NSCLC carrying EGFR amplifications. Numerous research studies have focused primarily on the relationship between $E G F R$ gene mutation and lung cancer progression and treatment. In the present study, we used fluorescence in situ hybridization (FISH) to detect the EGFR amplification status in patients with NSCLCs, and explored the relationship between EGFR amplification and NSCLC invasion and metastasis.

\section{MATERIAL AND METHODS}

\section{Tissue samples}

Lung tissue samples from 45 patients with NSCLC and 15 normal controlled samples were collected from lung biopsy and pathological diagnosis between December 2010 and August 2014 in The First People's Hospital of Jining City. All of the 45 patients with NSCLC underwent surgery without preoperative radiotherapy or chemotherapy. There were 25 men and 20 women in the NSCLC group and the average age was 53 years (40-72 years). Pathological types of NSCLC included 23 cases of squamous carcinoma and 22 cases of adenocarcinoma. Tumors were staged pathologically according to the Union International Contre le Cancer (UICC-7) staging system for lung cancer, including 10 cases in stage I, 17 in stage II, and 8 in stage III. Of the 45 patients with NSCLC, 20 appeared to have lymph node metastases. Written consents have been obtained from patients and their families. The research protocol has been approved by the Ethics Committee of our hospital.

\section{Reagents}

The EGFR gene amplification detection kit was purchased from the GPMedical Technology Company (Beijing, China). Proteinase K was obtained from TaKaRa Bio (Otsu, Shiga, Japan). An Olympus BX53 fluorescence microscope (Olympus, Tokyo, Japan) was used for fluorescence detection.

\section{Detection method}

Specimens were fixed with 10\% formaldehyde, embedded in paraffin, and sliced to 4 $\mu \mathrm{m}$. The slides were baked overnight and treated with proteinase $\mathrm{K}$. Slides were subsequently rinsed with $2 \mathrm{X}$ saline sodium citrate (SSC) solution and put in $-20^{\circ} \mathrm{C}$ precooling baths of 70,85 , and $70 \%$ ethanol for 2 min each. An EGFR FISH probe (10 pM) was synthesized using specific sequence complementary to EGFR mRNA nt 301-486 using a fluorescentlabeling in vitro transcription kit (Roche, Basel, Switzerland) and was applied on the tissue section hybridization area and the slides were hybridized as followed: $83^{\circ} \mathrm{C}$ co-degeneration for $5 \mathrm{~min}$, followed by $42^{\circ} \mathrm{C}$ hybridization for $4-16 \mathrm{~h}$. After hybridization, the slides were immediately put into $0.3 \% \mathrm{NP}-40 / 0.4 \mathrm{X}$ SSC solution for $30 \mathrm{~min}$ and then put into $0.1 \% \mathrm{NP}$ 40/2X SSC solution for another 30-min incubation, followed by a rinse in $70 \%$ ethanol and staining with $15 \mu \mathrm{L}$ DAPI. To work as an internal reference, centromere was also stained using fluorescent probes. Negative controls were performed using the sense-strand of the $E G F R$ gene as a probe. 


\section{Determination of results}

In the fluorescent images, a red signal represented the EGFR gene. FISH results were observed under a 100X objective and 100 cells were counted. The ratio was calculated as follows: Ratio $=$ total red signal in 100 nuclei / green signal in 100 cells). Positive judgment criteria: 1 ) Ratio $\geq 2 ; 2$ ) the number of cells with $\geq 15$ red signals accounted for more than $10 \%$ of the total cells; 3 ) appearance of clustered cells with positive signals; 4) Ratio $<2$, but the number of cells with $\geq 4$ red signals accounted for more than $40 \%$ of the total cells.

\section{Statistical analysis}

The SPSS19.0 (SPSS, Chicago, IL, USA) statistical software package was used for data analysis. The chi-square test was used for enumeration and data comparison. The Spearman method was used for correlation analysis. $\mathrm{P}<0.05$ was considered to be statistically significant.

\section{RESULTS}

\section{EGFR gene amplification in NSCLC tissue}

Of the NSCLC tissues, 29 in 45 appeared positive for $E G F R$ gene amplification, while no amplification was evident in the remaining 16 (Figure 1). The positive rates of $E G F R$ gene amplification in normal lung tissues and NSCLC tissues were 13.33 and $64.44 \%$, respectively $\left(\chi^{2}=11.77, \mathrm{P}<0.05\right)$
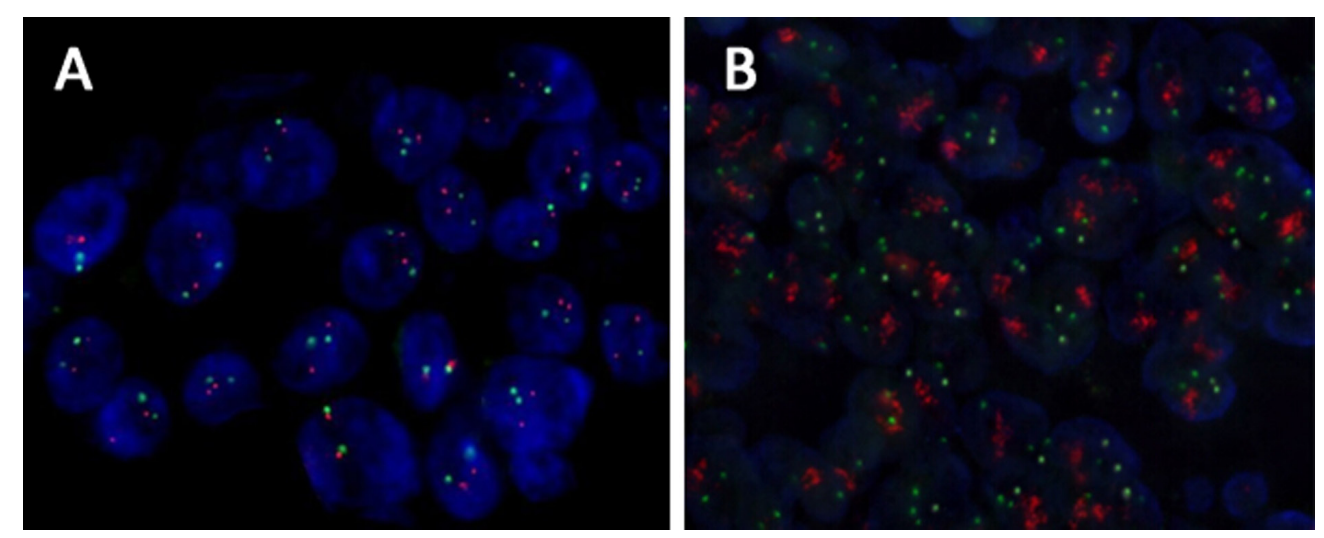

Figure 1. EGFR gene amplification in NSCLC tissue. A. Negative EGFR amplification in NSCLC tissue (100X, Ratio =1). B. Positive EGFR amplification in NSCLC tissue $(100 \mathrm{X}$, Ratio $>2)$. Red color indicates the positive signal of $E G F R$ gene; green color is the centromere; blue, DAPI. NSCLC = non-small cell lung cancer.

\section{EGFR gene amplification with NSCLC clinicopathologic characteristics}

There were significant differences in the rates of EGFR gene amplification between NSCLC and normal lung tissues $(\mathrm{P}<0.01$; Table 1). As shown in Table 2, the EGFR 
amplification-positive rate in patients aged $<60$ years was $66.67 \%(14 / 21)$, while it was $62.50 \%(15 / 24)$ in patients aged $\geq 60$ years. Male patients had a $64.0 \%(16 / 25)$ positive $E G F R$ gene amplification rate, while female patients had $65.0 \%(13 / 20)$. Histologically, the positive rates in squamous carcinoma and adenocarcinoma were 56.52\% (13/23) and $72.72 \%(16 / 22)$, respectively. These results suggested that the rate of $E G F R$ gene amplification did not correlate with patient age, gender, or histopathologic type $(\mathrm{P}>0.05)$. On the other hand, positive $E G F R$ amplification was more often observed in patients with lymph node metastasis $(90.0 \%)$ than in those without $(44.0 \%)(\mathrm{P}<0.05)$. The $E G F R$-positive amplification rate increased following the rise of NSCLC clinical stage $(\mathrm{P}<0.05)$. The positive rate in stage III tumors was significantly higher than that in stage II, while there was a lack of statistical difference between stages II and I. Finally, the positive amplification rate was lower in patients with moderate-poor differentiation than in well-differentiated ones $(\mathrm{P}<0.05)$.

Table 1. EGFR amplification in NSCLC and normal lung tissue.
\begin{tabular}{lccc}
\hline \multicolumn{3}{c}{ EGFR amplification } & P value \\
\cline { 2 - 4 } & Negative & Positive & 0.01 \\
\hline NSCLC & 16 & 29 & 2 \\
Normal lung tissue & 13 & 2 & \\
\hline TSCLC
\end{tabular}

NSCLC $=$ non-small cell lung cancer.

Table 2. EGFR gene amplification with NSCLC clinicopathologic characteristics.

\begin{tabular}{|c|c|c|c|c|}
\hline & \multirow[t]{2}{*}{ Patient } & \multicolumn{2}{|c|}{$E G F R$ amplification } & \multirow[t]{2}{*}{$P$ value } \\
\hline & & Negative & Positive (\%) & \\
\hline \multicolumn{5}{|l|}{ Age (years) } \\
\hline$<60$ & 21 & 7 & $14(66.67 \%)$ & \\
\hline$\geq 60$ & 24 & 9 & $15(62.50 \%)$ & 0.77 \\
\hline \multicolumn{5}{|l|}{ Gender } \\
\hline Male & 25 & 9 & $16(64.00 \%)$ & \\
\hline Female & 20 & 7 & $13(65.00 \%)$ & 0.94 \\
\hline \multicolumn{5}{|l|}{ Histologic type } \\
\hline Squamous carcinoma & 23 & 10 & $13(56.52 \%)$ & \\
\hline Adenocarcinoma & 22 & 6 & $16(72.72 \%)$ & 0.10 \\
\hline \multicolumn{5}{|l|}{ Lymph node metastases } \\
\hline Negative & 25 & 14 & $11(44.00 \%)$ & \\
\hline Positive & 20 & 2 & $18(90.00 \%)$ & $<0.01$ \\
\hline \multicolumn{5}{|l|}{ Differentiation } \\
\hline Moderate-well & 25 & 14 & $12(48.00 \%)$ & \\
\hline Poor & 20 & 3 & $17(85.00 \%)$ & $<0.01$ \\
\hline \multicolumn{5}{|l|}{ TNM staging } \\
\hline I & 10 & 7 & $3(30.00 \%)$ & \\
\hline II & 17 & 7 & $10(58.82 \%)$ & \\
\hline III & 18 & 2 & $16(88.89 \%)$ & $<0.01$ \\
\hline
\end{tabular}

$\mathrm{NSCLC}=$ non-small cell lung cancer; $\mathrm{TNM}=$ tumor, node, metastasis.

\section{DISCUSSION}

C-erb-1 is located in human chromosome 7 p11.2 and encodes EGFR. The C-erb-1 (EGFR) gene is approximately $200 \mathrm{~kb}$ and contains 28 exons (Baselga and Arteaga, 2005). The $E G F R$ gene encodes a type of glycoprotein receptor that locates on the surface of the 
cell membrane. This receptor contains an extracellular region, a transmembrane region, an intracellular region, and a carboxyl terminus, and has tyrosine kinase activity. Its activation can lead to an increase in DNA synthesis, and can stimulate cell proliferation by binding to its ligand (Baselga and Arteaga, 2005; Pinter et al., 2008). EGFR homotypes or heterotypes undergo dimerization after binding of the extracellular region to the ligand, which includes EGF and TGF $\alpha$. Following this change, the tyrosine kinase domain in the intracellular region becomes activated and stimulates cell proliferation.

It has been confirmed that EGFR is overexpressed in many tumors and is closely related to tumor development, invasion, and metastasis (Sun et al., 2015; Khambata-Ford et al., 2010). EGFR overexpression has been found in $60-80 \%$ of NSCLCs (Dacic et al., 2006). Since the EGFR receptor is necessary for the EGFR signaling pathway, EGFR receptor target therapy has become an important part of NSCLC treatment (Tsao et al., 2005). TKIs that block EGFR tyrosine kinase receptor activation or competitive monoclonal antibody drugs that interact with the extracellular ligand binding sites can prevent EGFR receptor signal transduction, further inhibiting tumor cell proliferation, invasion, metastasis, and angiogenesis, while also inducing tumor cell apoptosis to prevent tumor progression (Tang et al., 2005; Lee et al., 2010). At present, EGFR-TKIs have been used for NSCLC treatment clinically to considerable effect. In some patients, an EGFR mutation has been identified located at the ATP binding site of the tyrosine kinase activity domain. Mutation at this site allows for self-activation of the EGFR tyrosine kinase domain without the need for ligand binding, resulting in a stronger and constitutive proliferation signal. This has become the theoretical basis explaining the efficacy of EGFR-TKIs for the treatment of NSCLCs with EGFR mutations. Han et al. (2006) has suggested that EGFR mutation is the independent variable for the TKI curative effect after studying the relationship between $E G F R$ gene mutation, positive $E G F R$ amplification, and $K R A S$ mutation with long-term survival of patients with NSCLC in East Asia. On the other hand, although patients with NSCLC have shown good response to the TKIs, the latter cannot significantly prolong survival. These results suggest the conclusion that numerous EGFR receptors can enhance EGFR signaling pathways following ligand binding when large amounts of EGFR are present through amplification or overexpression. This mechanism might have a crucial role in the occurrence, progression, and metastasis of NSCLCs. However, the relationships between EGFR amplification status and the clinical curative effect of EGFRTKIs have not been well reported (Xia et al., 2011). The present study focused on EGFR gene amplification in NSCLC pathogenesis, and investigated its correlation with clinical pathologic factors. EGFR amplification status was measured by FISH in 45 NSCLC and 15 normal lung tissues, and the positive rate in the former was $64.44 \%$, which was consistent with previously published research (Sun et al., 2015). Our results showed that the positive EGFR gene amplification rate had no relationship with patient age and gender, which was also in line with previous study results (Pirker et al., 2012). Patients with NSCLC and lymph node metastasis presented higher EGFR gene amplification rates; in addition, the EGFR gene amplification rate increased following TNM upstaging of the cancers. These results indicated that EGFR gene amplification might be closely associated with NSCLC invasion and metastasis, and also with NSCLC progression.

Although the sample size is small in our study, the preliminary results suggested that NSCLC had a relative high rate of EGFR gene amplification regardless of the metastasis status. On the other hand, the EGFR gene amplification rate was associated with the degree of differentiation of the NSCLCs, a finding that is worth further study. 


\section{Conflicts of interest}

The authors declare no conflict of interest.

\section{REFERENCES}

Baselga J and Arteaga CL (2005). Critical update and emerging trends in epidermal growth factor receptor targeting in cancer. J. Clin. Oncol. 23: 2445-2459.

Cappuzzo F, Hirsch FR, Rossi E, Bartolini S, et al. (2005). Epidermal growth factor receptor gene and protein and gefitinib sensitivity in non-small-cell lung cancer. J. Natl. Cancer Inst. 97: 643-655.

Dacic S, Flanagan M, Cieply K, Ramalingam S, et al. (2006). Significance of EGFR protein expression and gene amplification in non-small cell lung carcinoma. Am. J. Clin. Pathol. 125: 860-865.

Han SW, Kim TY, Jeon YK, Hwang PG, et al. (2006). Optimization of patient selection for gefitinib in non-small cell lung cancer by combined analysis of epidermal growth factor receptor mutation, K-ras mutation, and Akt phosphorylation. Clin. Cancer Res. 12: 2538-2544.

Jeon YK, Sung SW, Chung JH, Park WS, et al. (2006). Clinicopathologic features and prognostic implications of epidermal growth factor receptor $(E G F R)$ gene copy number and protein expression in non-small cell lung cancer. Lung Cancer 54: 387-398.

Jorissen RN, Walker F, Pouliot N, Garrett TP, et al. (2003). Epidermal growth factor receptor: mechanisms of activation and signalling. Exp. Cell Res. 284: 31-53.

Khambata-Ford S, Harbison CT, Hart LL, Awad M, et al. (2010). Analysis of potential predictive markers of cetuximab benefit in BMS099, a phase III study of cetuximab and first-line taxane/carboplatin in advanced non-small-cell lung cancer. J. Clin. Oncol. 28: 918-927.

Lee HJ, Xu X, Choe G, Chung DH, et al. (2010). Protein overexpression and gene amplification of epidermal growth factor receptor in nonsmall cell lung carcinomas: Comparison of four commercially available antibodies by immunohistochemistry and fluorescence in situ hybridization study. Lung Cancer 68: 375-382.

Lynch TJ, Bell DW, Sordella R, Gurubhagavatula S, et al. (2004). Activating mutations in the epidermal growth factor receptor underlying responsiveness of non-small-cell lung cancer to gefitinib. N. Engl. J. Med. 350: 2129-2139.

Paez JG, Jänne PA, Lee JC, Tracy S, et al. (2004). EGFR mutations in lung cancer: correlation with clinical response to gefitinib therapy. Science 304: 1497-1500.

Parkin DM, Bray F, Ferlay J and Pisani P (2005). Global cancer statistics, 2002. CA Cancer J. Clin. 55: 74-108.

Pinter F, Papay J, Almasi A, Sapi Z, et al. (2008). Epidermal growth factor receptor (EGFR) high gene copy number and activating mutations in lung adenocarcinomas are not consistently accompanied by positivity for EGFR protein by standard immunohistochemistry. J. Mol. Diagn. 10: 160-168.

Pirker R, Pereira JR, von Pawel J, Krzakowski M, et al. (2012). EGFR expression as a predictor of survival for first-line chemotherapy plus cetuximab in patients with advanced non-small-cell lung cancer: analysis of data from the phase 3 FLEX study. Lancet Oncol. 13: 33-42.

Rivera MP (2004). Multimodality therapy in the treatment of lung cancer. Semin. Respir. Crit. Care Med. 25: 3-10.

Sibilia M, Kroismayr R, Lichtenberger BM, Natarajan A, et al. (2007). The epidermal growth factor receptor: from development to tumorigenesis. Differentiation 75: 770-787.

Stinchcombe TE and Socinski MA (2010). Targeted therapies: Biomarkers in NSCLC for selecting cetuximab therapy. Nat. Rev. Clin. Oncol. 7: 426-428.

Sun G, Liu B, He J, Zhao X, et al. (2015) Expression of EGFR Is Closely Related to Reduced 3-Year Survival Rate in Chinese Female NSCLC. Med. Sci. Monitor: Int. Med. J. Exp. Clin. Res. 21: 2225-2231.

Tamura K, Okamoto I, Kashii T, Negoro S, et al. (2008). Multicentre prospective phase II trial of gefitinib for advanced non-small cell lung cancer with epidermal growth factor receptor mutations: results of the West Japan Thoracic Oncology Group trial (WJTOG0403). Br. J. Cancer 98: 907-914.

Tang X, Shigematsu H, Bekele BN, Roth JA, et al. (2005). EGFR tyrosine kinase domain mutations are detected in histologically normal respiratory epithelium in lung cancer patients. Cancer Res. 65: 7568-7572.

Tsao MS, Sakurada A, Cutz JC, Zhu CQ, et al. (2005). Erlotinib in lung cancer - molecular and clinical predictors of outcome. N. Engl. J. Med. 353: 133-144.

Xia LP, Qiu HJ, Chen XX, Hu PL, et al. (2011). Short-term outcomes of cetuximab combined with standard chemotherapy as first line setting for Chinese patients with non-small cell lung cancer: a report of 12 cases. Med. Oncol. 28: S570-576.

Zhang ZH, Yu M, Ni CC and Lu YJ (2010). The expression of EGFR in non-small cell lung cancer and its clinical significance. J. Clin. Pulm. Med. 15: 1079-1081. 